\title{
Design of equi-interference lines in CDMA mobile systems
}

\author{
Aleksandar Lebl*, Dragan Mitićc* ${ }^{* *}$ Žarko Markov* Željka Tomićc***
}

\begin{abstract}
In this paper it is presented the process of designing equi-interference lines in the CDMA mobile telephony systems. It is proved that shape of these lines in one base station cell is determined by emission characteristics of that base station and the base stations, which form the first ring around the considered cell. The influence of base stations from the other rings after the first one is dominantly noticed as the interference increase in each point of equi-interference line. The value of interference increase depends on the value of environmental propagation coefficient. For its small values it is necessary to consider the influence of base stations from more than twenty rings around the analyzed cell, while for great values it is enough to consider only two rings.
\end{abstract}

K e y w or ds: base station cell, equi-interference lines, environmental propagation coefficient, rings of base stations

\section{Introduction}

Mobile communications occupy more and more space in our lives. The technology of these communications progresses, but the problems, with which it is coping in the development process, remain mainly the same. Among these problems interference is one of the major challenges.

The factors which contribute to interference increase are numerous [1]. The main reason for such problems is the existence of multiple spatially distributed base stations (BTSs), forming a mobile network. The signal from these BTSs, although attenuated due to wireless propagation, may not be neglected when considering the transmitted signal quality in the analyzed BTS cell. The presence of large objects on the path between mobile signal transmitter and receiver causes shadowing and slow fading. These objects may also lead to signal reflection. As a consequence, fast fading and multipath fading appear. One additional detailed survey of factors, which are the cause of interference increase, is presented in [2]. As the number of implemented different wireless systems constantly grows, the problem of their coexistence in the same frequency bands and interference increase becomes every day more sever [3].

Interference influence is very complex for the analysis and it is difficult to find the optimal model, valid for various systems of wireless signal transmission. For example, model presented in [4] is designed for multiple-input multiple-output wireless local area network (MIMOWLAN). There is a number of contributions where attention is devoted to the choice of interference model, as, for example, in $[5,6]$. But, these models in $[5,6]$ do not correspond to mobile telephony systems analysis.
The dominant source of interference in mobile systems is the signal intended for other mobile users (mobile stations - MSs), which exists in the same frequency band and in the same time as the signal of the considered MS. There is a difference in the way how this interfering signal influences signal transmission in GSM $(2 \mathrm{G})$ and GSM like systems (GPRS, EDGE), on one side, and CDMA (3G) systems $[7,8]$, on the other side. GSM and GSM like systems are based on the implementation of frequency (FDMA) and time (TDMA) multiplex. It means that only a small part of frequency spectrum and in precisely defined time interval is reserved for one MS. The active MSs from the same mobile cell are separated in time or in frequency, or both in time and frequency. It is possible that MS from some of the surrounding cell coincide in time and frequency domain. Special attention is paid to the frequency band selection in each mobile cell to avoid the same frequency application in adjacent cells. As, so, the interference source is more than one ring distant from the considered MS, this disturbing signal is significantly attenuated. The conclusion of this short analysis is that in GSM and GSM like systems interference signal has a lower power than the regular signal. The typical values of signal-to-interference ratio $(S / I)$ are at least $17 \mathrm{~dB}$. It means that in GSM and GSM like systems interference is not an important problem source.

In CDMA systems situation is different. Each MS uses the whole predicted frequency band, which is intended for these systems $[9,10]$. Therefore, all active MSs in the same cell as the analyzed MS (excluding this analyzed MS) and active MSs from adjacent cells cause interference. Interference is significantly greater than regular signal: the value of $S / I$ may be in the worst case between $-15 \mathrm{~dB}$ (when taking into account only the MSs from the same

\footnotetext{
* Department of Radio Communications Engineering, Institute IRITEL a.d., Belgrade 11080, Serbia, lebl@iritel.com, zmarkov@iritel.com, ** Department of Optical Communications, Institute IRITEL a.d., Belgrade 11080, Serbia, mita@iritel.com, *** Tehnikum Taurunum College of Applied Engineering Studies in Belgrade, 11080, Serbia, ztomic@tehnikum.edu.rs
} 
cell) and $-17 \mathrm{~dB}$ (when taking into account also the MSs from the adjacent cells). The similar calculation process is implemented for LTE (4G) [11] and 5G systems [12]. A brief review of the interference influence when MSs are moving in the cell and over the cell rim leading to handover may be found in [13].

As it may be concluded from this brief survey, this multiple access interference (MAI) is a great problem in mobile communications, especially in CDMA systems. Although coding algorithm in CDMA systems is designed in such a way that one interfering user has small effect on the reception of regular signal, the total influence of all interferers becomes significant [14]. The problem of MAI influence may be overcome in future by the implementation of Interleave Division Multiple Access (IDMA) systems [15].

In this paper we present the method for designing the lines of equal interference (equi-interference lines) in mobile telephony systems. The characteristics of some parameter equal value are often implemented in different scientific fields. For example, such characteristics are introduced when analyzing quality of VoIP connections. The equi-quality characteristics in this case may be lines (in two dimensions), when contribution of packet delay and packet loss on voice quality is analyzed [16] or surfaces (in three dimensions) when mutual influence of packet delay, packet loss and echo is analyzed [17]. These characteristics are very useful for planning Internet voice connections.

The purpose of equi-interference lines in mobile system networks is just to model interference in each point of BTS cell. These lines are different for different types of mobile systems (GSM, CDMA, etc). In the case of GSM systems adjacent BTSs do not contribute to the interference, because they operate in different frequency band than the considered BTS. Only BTSs two or more cell area distant are in the same frequency band and contribute to the interference and to the designed interference lines. On the contrary, in CDMA systems all BTSs operate in the same frequency band and are the source of interference.

\section{Model, assumptions and designations}

Let us suppose that it is necessary to calculate interference level in CDMA network in some point A, whose coordinates are $(x, y)$, Fig. 1 . For this analysis it is important to emphasize that MS is placed in point $\mathrm{A}$. The location of the point is in the hexagonal cell 0 , which is controlled by the base station $\mathrm{BTS}_{0}$. Here, $\mathrm{BTS}_{0}$ is situated in the centre of the cell and its coordinates are $(0,0)$. The distance between point $\mathrm{A}$ and $\mathrm{BTS}_{0}$ is, then

$$
r_{0}=\sqrt{x^{2}+y^{2}}
$$

The interference level in the point $\mathrm{A}$ is not influenced only by $\mathrm{BTS}_{0}$, but also by other BTSs in the mobile system network. Let us consider one of these BTSs, $\mathrm{BTS}_{i}$.
The coordinates of $\mathrm{BTS}_{i}$ are $\left(x_{i}, y_{i}\right)$. The distance between point $\mathrm{A}$ and $\mathrm{BTS}_{i}$ may be determined as

$$
r_{i}=\sqrt{\left(x_{i}-x\right)^{2}+\left(y_{i}-y\right)^{2}} .
$$

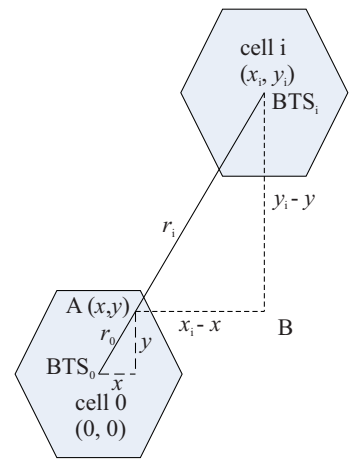

Fig. 1. Calculation of distance between some point $\mathrm{A}$ and base station

The model of mobile network, which is used in our analysis, is the usually used model, consisted of a number of hexagonal BTS cells. The goal is to determine total interference in a point $(x, y)$ in a central cell, Fig. 2. The other cells in mobile network may be considered as forming rings around the central cell. Each cell in Fig. 2 (except the central cell) is designated by two indices: the first one is the ordinary number of the ring around the central cell and the second one is the ordinary number of the cell in the considered ring of BTS cells. The coordinates of each BTS are presented by two numbers in brackets, where the value 1 corresponds to the length of hexagon edge, ie cell radius.

Let us now suppose that the total number of cell rings is $\mathrm{n}$. These rings are designated by their ordinary numbers $j=1,2, \ldots$ The total number of BTSs in $n$ rings $(N)$ is now

$$
N=6 \sum_{j=1}^{n} j
$$

and the number of BTSs in the ring $j$ is $6 j$. The ordinary numbers of the BTSs in the ring $j$ are determined on the base of inequality

$$
1+6 \sum_{k=0}^{j-1} k \leq i \leq 6 \sum_{k=0}^{j} k .
$$

We suppose in our model that emission power of each BTS is the same, ie

$$
P_{0}=P_{1}=\cdots=P_{N}=P
$$

where $P_{0}$ is the emission power of central cell and $P_{1}, \ldots, P_{n}$ are the powers of BTSs in the analyzed rings.

The power which is transmitted from BTS to some MS (and also to the user in point $\mathrm{A}$ in the area of cell 0 ) is 


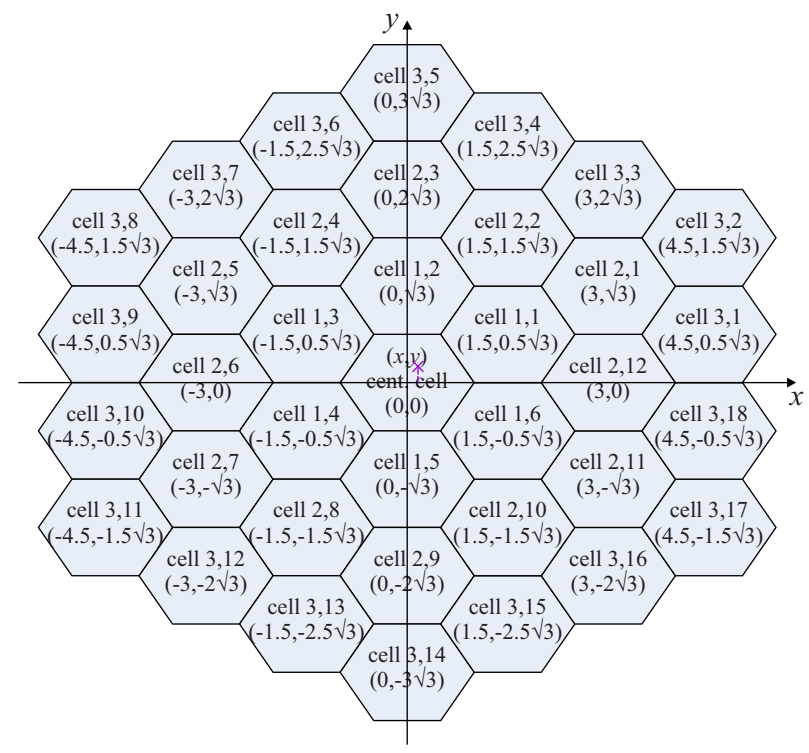

Fig. 2. The model of mobile network, used to calculate interference

adjusted according to the mutual distance BTS-MS. It may be expressed as

$$
P_{0}=a r_{0}^{\gamma}
$$

where $a$ is coefficient of proportionality, $r_{0}$ is the mutual distance $\mathrm{BTS}_{0}-\mathrm{MS}$ and $\gamma$ is environmental propagation coefficient. The value of $\gamma$ is $2 \leq \gamma \leq 5$, [18].

When considering the power of $\mathrm{BTS}_{0}$, it may be said that BTS emission power intended for all active MSs situated in the area of $\mathrm{BTS}_{0}$ cell except the power transmitted to user in point $\mathrm{A}$ contributes to the interference in point $\mathrm{A}$. The corresponding mathematical expression for the interference is $[10]$

$$
I_{0 c}=\alpha\left(P_{0}-P_{c}\right) r_{0}^{-\gamma} \approx \alpha P_{0} r_{0}^{-\gamma}
$$

where it is defined that $P_{c}$ is emission power intended only for the user in point $\mathrm{A}$ and $P_{0} \gg P_{c}$.

The total power of each other BTSs around the BTS where the considered MS is situated contributes to interference in point $\mathrm{A}$. The interference, caused by the emission power of $\mathrm{BTS}_{i}$, is

$$
I_{i c}=\alpha P_{i} r_{i}^{-\gamma}
$$

The total interference in the point $\mathrm{A}$ is now

$$
I_{c}=I_{0 c}+\sum_{i=1}^{N} I_{i c},
$$

on the base of (1), (2) and (9)

$$
I_{c}=\alpha P\left[\left(x^{2}+y^{2}\right)^{-\frac{\gamma}{2}}+\sum_{i=1}^{N} S\left(x_{i}, y_{i}\right)\right]
$$

where

$$
S\left(x_{i}, y_{i}\right)=\left[\left(x_{i}-x\right)^{2}+\left(y_{i}-y\right)^{2}\right]^{-\frac{\gamma}{2}}
$$

\section{The presentation of equi-interference lines}

The value of interference in each point in the $\mathrm{BTS}_{0}$ area may be calculated by (10). In order to design an equiinterference line, we choose all points of equal $I_{c}$ and connect them.

Figure 3 presents equi-interference lines, which are determined on the base of the influence of $\mathrm{BTS}_{0}$ and $\mathrm{BTS}_{1}$ BTS $_{6}$ (BTS from central cell and BTSs from the first ring). The lines are designed for the value $\gamma=3$. The value for each interference line is determined as the multiple of interference in points at unity distance from BTS when only interference caused by that, central BTS, is considered.

The borders between cell hexagons are also presented in Fig. 3. In the vicinity of some BTS influence of this BTS is dominant and here equi-interference lines are concentric circles with the centre in BTS. The maximum interference is in the BTS proximity. The value of interference decreases as faster as equi-interference line is nearer to BTS. In the case that equi-interference line is near the border between two cells, the shape of these lines is changed, because there is no dominant source of interference. The centre of these part of equi-interference lines is in the common point of three adjacent BTSs, where interference has its minimum.

Figure 4 presents equi-interference lines for various values of propagation coefficient $\gamma$. The lines are presented for two values of interference: one greater, which generates lines nearer to BTS and the other, which generates lines around the borders between BTS cells. The first lines are concentric circles with the centre in corresponding BTS, while the second lines have centre in the common point of three adjacent cells or very near to this point.

It is also important to determine how equi-interference lines are changed when new rings of BTSs are included in the calculation. It is clear that influence of rings of BTSs is as smaller as they are more distant from the considered BTS cell. From Fig. 3 and 4 it has been possible to conclude that BTSs in the first ring change the shape of equi-interference lines near the BTS cell borders.

Figure 5 presents two representative equi-interference lines: the first one when the influence of the first ring of BTSs is considered and the second one when the first and the second ring of BTSs are included in the analysis. The shape of these two lines is approximately the same. But, they differ in the relative value of interference: the first presented line (when there is one ring of BTSs) is for interference level 3.8 and the second one (when there are two rings of BTSs) is for interference level 4.3. The equi-interference lines are representative for the analysis, 


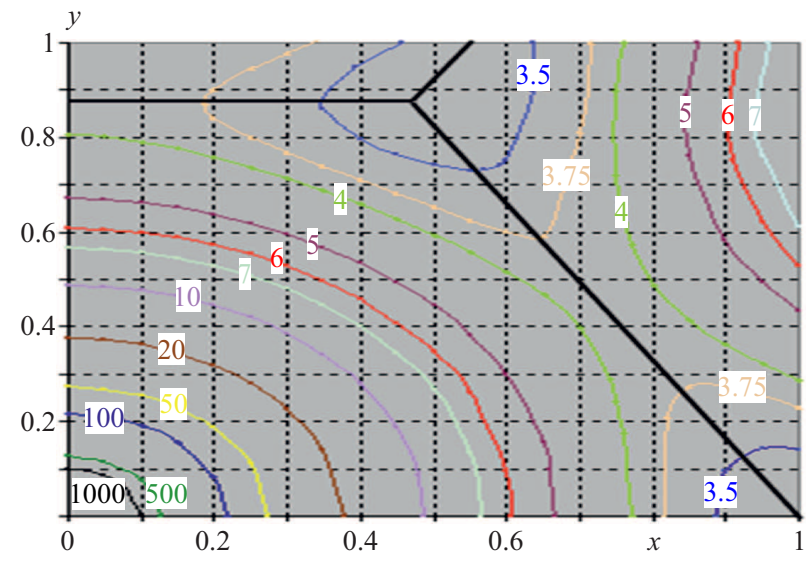

Fig. 3. Equi-interference lines for $\gamma=3$

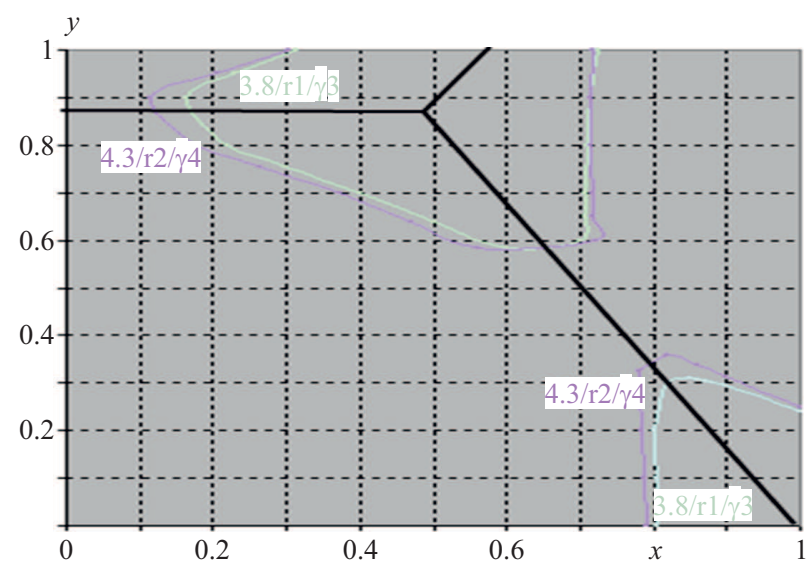

Fig. 5. Equi-interference lines under the influence of the first ring of BTSs and the first and the second ring of BTSs

because they illustrate interference near the cell borders, where the influence of surrounding BTSs is greatest.

\section{Contribution of further rings to interference value}

The goal of our further analysis has been to determine the influence of further BTSs rings (after the first ring) on the interference level. The contribution of further rings is calculated for the points in BTS area, which have been used to form equi-interference lines according to Figures 3 and 4. On the base of (2), (4) and (10) this relative contribution in percent for some ring $j, j \geq 2$, is in a point $(x, y)$

$$
\begin{gathered}
\Delta I_{j c}=100 \frac{\sum_{l o}^{h i} S\left(x_{i}, y_{i}\right)}{\left(x^{2}+y^{2}\right)^{-\frac{\gamma}{2}}+\sum_{1}^{6} S\left(x_{i}, y_{i}\right)} \\
\text { where } \quad l o=1+6 \sum_{k=0}^{j-1} k, h i=6 \sum_{k=0}^{j} k
\end{gathered}
$$

and $S\left(x_{i}, y_{i}\right)$ is given by $(10)$.

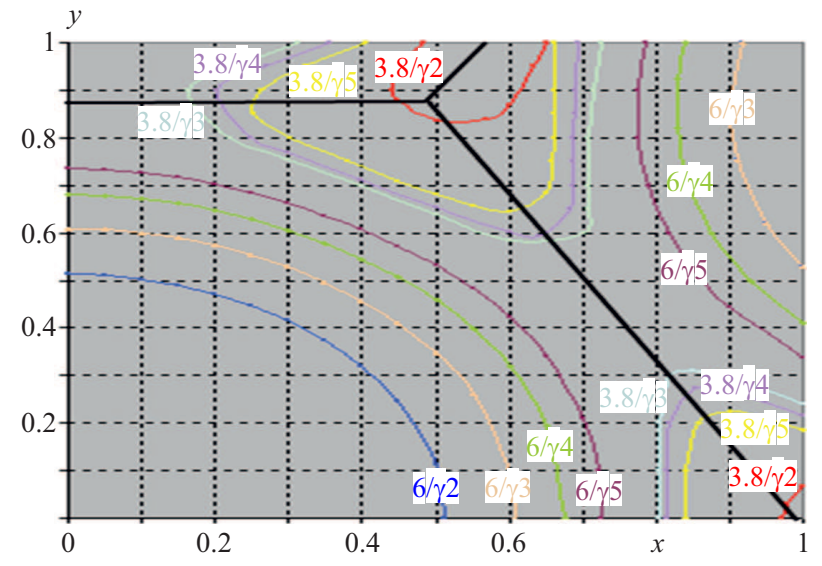

Fig. 4. Equi-interference lines as a function of $\gamma$

Figures 6,7 and 8 present relatively, in percent, minimum and maximum contribution of BTSs situated in rings 2 to 6 to the interference value caused by the BTSs in central cell and in the first ring of cells. The results are calculated according to (11). The points, which are chosen to calculate the contribution of further rings to the interference, form equi-interference line of the relative value 3.8. This line is located near the border of the cell, which means that interference caused by BTS in central cell is not too great. The contribution is calculated for $\gamma=2$ (Fig. 6), $\gamma=3$ (Fig. 7) and $\gamma=4$ (Fig. 8).

The calculation, which is illustrated by the graphs in Figures 6, 7 and 8, proves that BTSs in central cell and in the first ring mainly contribute to the interference in the central cell. When considering further rings of BTSs, it is obvious that influence of these BTSs is decreasing when the value of $\gamma$ increases. Relative difference between minimum and maximum influence on interference is small: even in the worst case it is less than $3 \%$ of the interference caused by BTSs in central cell and the first ring. That is why it can be adopted that the shape of equi-interference lines does not change when new rings of BTSs after the ring 1 are included in the consideration. Only the relative measure for each equi-interference line is increased in that situation. This measure is increased in accordance to the graphs in Figures 6, 7 and 8. For example, if we adopt that three rings of BTSs are considered in the interference level determination, we take the values of interference for equi-interference lines from Fig. 3 or Fig. 4 and add the mean interference increase value $\Delta I$ separately for the ring 2 and ring 3 . Both values of $\Delta I$ are determined on the base of mean percent of relative interference increase $\left(\Delta I_{j c}\right)$ between its minimum and maximum value, depending on the value of $\gamma$. The corresponding value of $\Delta I_{j c}$ is selected from one of the Figures 6,7 or 8 . As the results, we obtain the interference value for equi-interference lines when rings 2 and 3 of BTSs are included in the analysis.

Figure 9 presents the value of interference when new rings of BTSs are included in the calculation of interference level, for values of $\gamma$ from 2 to 5 . This value of 


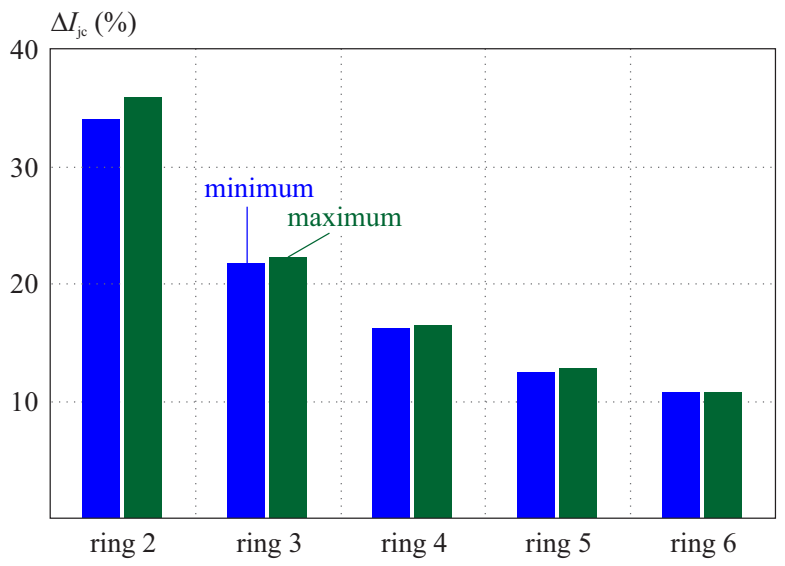

Fig. 6. Maximum and minimum contribution of rings 2 to 6 (in \%) to total interference caused by central cell and the first ring of BTSs when it is $\gamma=2$

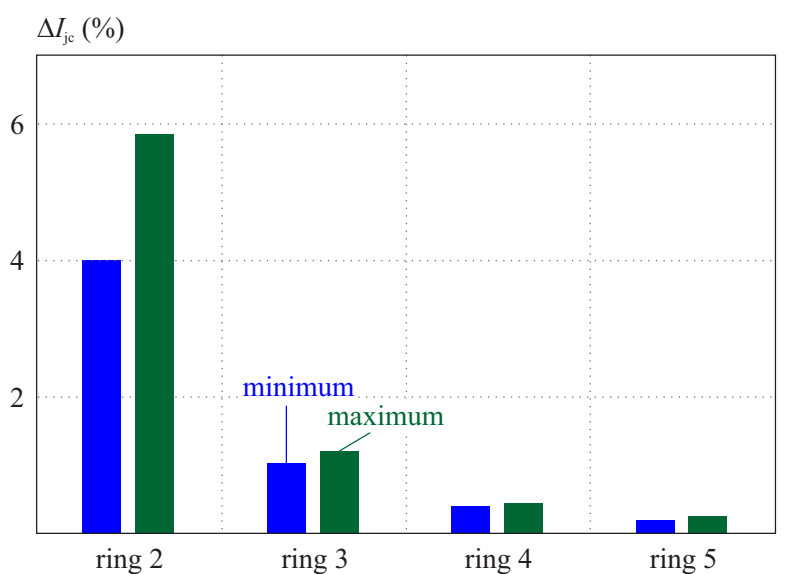

Fig. 8. Maximum and minimum contribution of rings 2 to 6 (in \%) to total interference caused by central cell and the first ring of BTSs when it is $\gamma=4$

interference is presented for the common point of three adjacent cells. The results are presented for first 25 rings.

Figure 10 is related to Fig. 9. It presents the relative increase of interference value when each new ring of BTSs is included in the calculation, again for various values of $\gamma$. This graph may be used to determine how many rings of BTSs have to be encountered in the consideration if we want that interference increase is smaller than some limit value after involving each new ring of BTSs in the

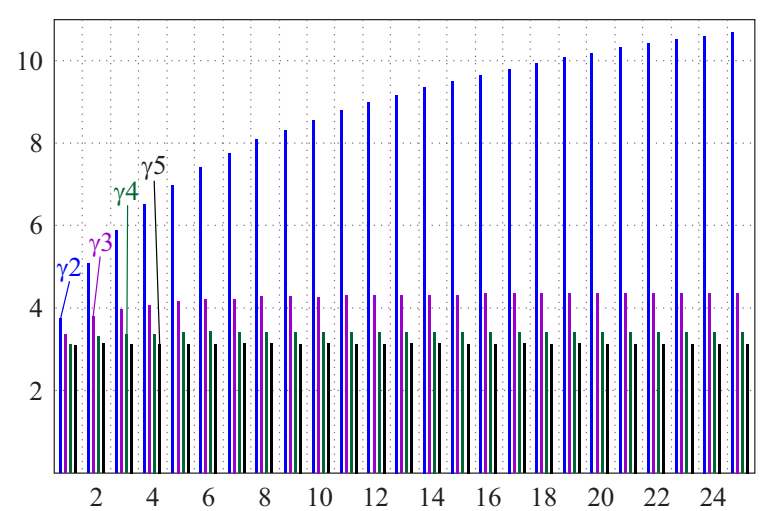

Fig. 9. Relative value of total interference when new rings (2 to 25) of BTSs are included in the calculation for $\gamma$ from 2 to 5

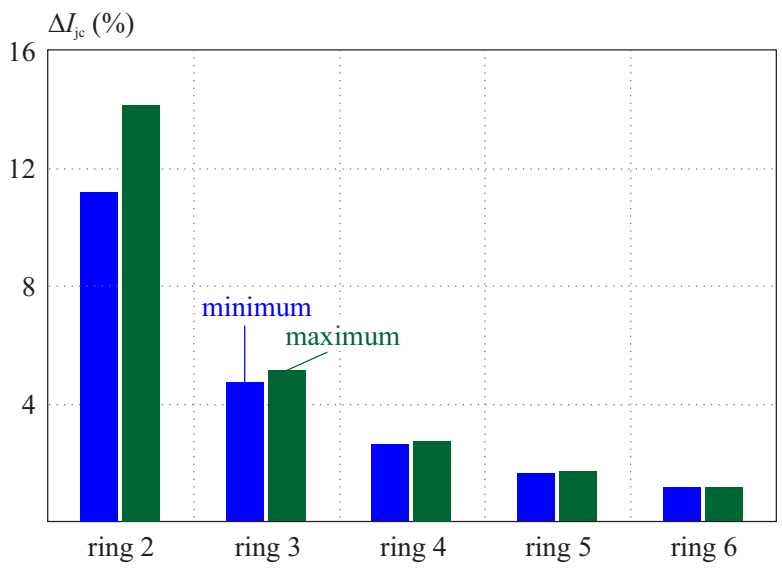

Fig. 7. Maximum and minimum contribution of rings 2 to 6 (in \%) to total interference caused by central cell and the first ring of BTSs when it is $\gamma=3$

calculation. For example, if this limit value of interference increase is $1 \%$, it is necessary to consider 23 rings when it is $\gamma=2$, but only 6 rings when $\gamma=3,3$ rings when $\gamma=4$ and 2 rings when $\gamma=5$. This calculation may be considered as the amendment to the corresponding statement from [10] about the number of rings of BTSs, which contribute to the interference.

\section{Conclusion}

Level of interference in the area of BTS cell is very important for mobile systems correct function. In the vicinity of BTS interference is mainly determined by the emission of that BTS, while near the BTS border interference is also significantly determined by the emission of the first BTSs ring around the analyzed cell. The level of interference decreases when the distance from BTS increases.

The purpose of equi-interference lines is to model the interference in the area of BTS cell. Equi-interference lines have the shape of concentric circles around BTS with the maximum in the BTS proximity, while near the BTS border these lines have minimum in the common point of three adjacent cells.

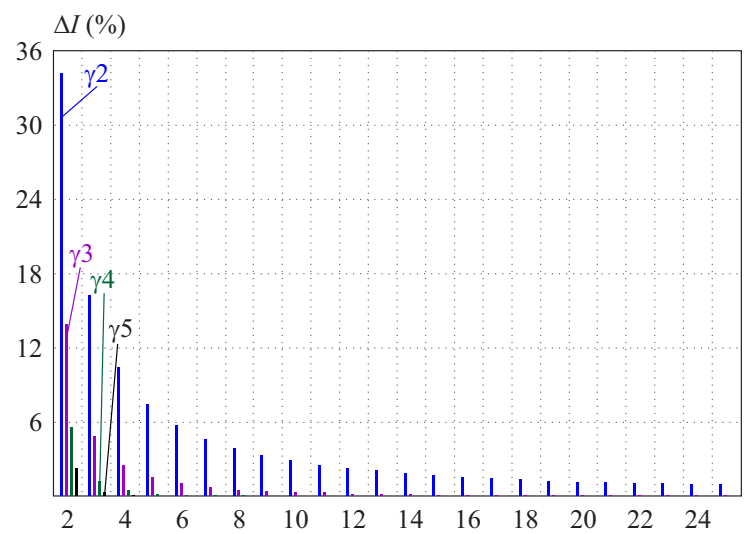

Fig. 10. Relative increase of total interference when new rings $(2$ to 25) of BTSs are included in the calculation for $\gamma$ from 2 to 5 
The other rings of BTSs starting from the second one only increase the level of interference, but nearly have no influence on equi-interference lines shape. The number of rings, which have to be taken into account when interference is analyzed, significantly depends on the value of $\gamma$. It is greater than 20 for $\gamma=2$ and only 2 for $\gamma=5$ if the goal is to limit the contribution of BTSs from one ring at interference increase less than $1 \%$.

\section{Acknowledgements}

The authors thank to Prof Ivo Kostić for very useful advices in paper improvement.

\section{REFERENCES}

[1] L. Irio, D. Oliveira, and R. Oliveira, "Interference Estimation in Wireless Mobile Random Waypoint Networks", Telfor Journal vol. 8, no. 2, pp. 93-97, Nov 2016.

[2] M. Grötschel, "Frequency Assignment in Mobile Phone Systems", ZIB-Report 00-58 Konrad-Zuse-Zentrum fr Informationtechnik Berlin, pp. 1-7, Dec 2000.

[3] Z. Nikolić, N. Milošević, M. Tošić, V. Nejković, and F. Jelenković, "Wireless Networks Coexistence in Unlicenced Bands", International Journal of Electrical Engineering Computing vol. 1, no. 1, pp. 1-10, Dec 2017.

[4] C. T. Hsieh, S. H. Liao, C. C. Chiu, and M. H. Ho, "Capacity Analysis of MIMO-WLAN Systems with Single Co-Channel Interference", Automatika vol. 55, no. 2, pp. 143-152, June 2014.

[5] A. Iyer, C. Rosenberg, and A. Karnik, "What is the Right Model for Wireless Channel Interference?", IEEE Transactions on Wireless Communications vol. 8, no. 5, pp. 2662-2671, May 2009.

[6] H. Shokri-Ghadikolaei, C. Fischione, and E. Modiano, "On the Accuracy of Interference Models in Wireless Communications", IEEE International Conference on Communications (ICC) 2016 pp. 1-7, 23-27 May 2016.

[7] A. Mitra, "Lecture Notes on Mobile Communication", Department of Electronics communication Engineering Indian Institute of Technology Guwahati, India,, pp. 23-53, Nov 2009.

[8] D. Tse and P. Wiswanath, "Fundamentals of Wireless Communication", Cambridge University Press 2005, pp. 120-165.

[9] D. Zhao, "Power Distribution Performance Analysis for Wireless Communication Networks", Springer 2012, pp. 17-37.

[10] M. S. Ismail and T. A. Rahman, "Forward-Link Performance of CDMA Cellular System", IEEE Transactions on Vehicular Technology vol. 49, no. 5, pp. 1692-1696, Sep 2000.

[11] N. A. Ali, H. A. Mourad, H. M. Elsayed, M. El-Soudani, H. H. Amer, and R. M. Daoud, "General Expressions for Downlink Signal to Interference Noise Ratio in Homogeneous Heterogeneous LTE - Advanced Networks", Journal of Advanced Research vol. 7, pp. 923-929, Sep 2016.

[12] M. Shoukat, B. S. Khan, S. Jangsher, A. Habib F, and A. Bhatti, "Resource Efficient Power Allocation in Small Cell Network" to appear in Physical Communication 2018, https://doi.org10.1016j.phycom.2018.07.014.

[13] A. Lebl, D. Mitić, B. Trenkić, and Ž. Markov, "Determination of Base Station Emission Power Change in a Mobile Network Cell with Movable Users", Radioengineering vol. 27, no. 4 Dec 2018.

[14] S. J. Honade and P. V. Ingole, "Removal of Multiple Access Interference in DS-CDMA Systems", International Journal of Scientific Research Publications vol. 2, no. 6, pp. 1-6, June 2012.

[15] A. Shukla and V. K. Deolia, "Performance Analysis of Modified Tent Map Interleaver in IDMA Systems", Journal of Electrical Engineering vol. 68, no. 4, pp. 318-321, Aug 2017.
[16] M. Mileusnić, A. Lebl, D. Mitić and Ž Markov, "About Delay Loss Equiquality Characteristics in Packet Telephony", $A u-$ tomatika vol. 55, no. 1, pp. 64-68, Feb 2014.

[17] A. Lebl, D. Mitić, P. Petrović, V. Matić, M. Mileusnić, and Ž. Markov, "The Application of Equal Quality Characteristics: Delay-Echo-Packet Loss to Internet Voice Connection Planning", 15th International Symposium INFOTEH Jahorina 6-18 III 2016, pp. 284-289.

[18] J. Ebersprächer and H. J. Vögel C. Bettstetter, GSM Switching Services Protocols, John Wiley \& Sons, second edition, 2001.

Received 11 December 2019

Aleksandar Lebl was born in Zemun, Serbia, in 1957. He received his BSc and MSc from the Faculty of Electrical Engineering in Belgrade, Republic of Serbia, in 1981. and 1986., respectively, and his PhD from the Faculty of Technical Science in Novi Sad, in 2009. He was employed from 1981 in the Switching Department of Institute for Electronics and Telecommunications IRITEL in Belgrade. During years he worked on the project of Digital Switching System for Serbian Telecommunication Industry. From 2015. he works in Radio communications department, also in IRITEL.

Dragan Mitić was born in Belgrade, Serbia, in 1953. He received his BSc and MSc from the Faculty of Electrical Engineering in Belgrade, Republic of Serbia, in 1977 and 1984, respectively, and his PhD from the Faculty of Technical Science in Novi Sad, in 2002. He is a senior research associate in IRITEL, Institute for Electronics and Telecommunications, Belgrade, Serbia. From 1977. until 1989. he was employed at the Land Forces Military Technical Institute in Belgrade, and since 1989. in IRITEL. Dr Mitić is author or co-author of more than 160 international and national scientific and professional papers. He works on several research projects for equipment of specific applications.

Žarko Markov was born in Žitište, Serbia, in 1946. He received his BSc, MSc and PhD from the Faculty of Electrical Engineering in Belgrade, Republic of Serbia, in 1969., 1975. and 1976., respectively. He is a scientific counsellor in IRITEL, Institute for Electronics and Telecommunications, Belgrade, Serbia. Area of work: Switching technics, Teletraffic theory, Network signalling. Author or co-author of more than hundred papers and six books. At the University of Belgrade, School of Electrical Engineering, Dr. Markov was a professor at the course of Switching technics and Network signalling.

Željka Tomič was born in Slavonski Brod, Croatia, in 1960. She received her BSc, MSc and PhD from the Faculty of Electrical Engineering in Belgrade, Republic of Serbia, in 1986, 1995 and 2007, respectively. She is a Research Assistant in IRITEL, Institute for Electronics and Telecommunications, Belgrade, Serbia. From 1987. until 2014. she was employed in IRITEL. Dr Tomić is a professor at the courses of Physics and Electrotechnics in Tehnikum Taurunum - College of Applied Engineering Studies in Belgrade, since 2011. She works on several research projects for equipment of specific applications, Laser beam interactions with materials of a different nature (ceramics type materials, ferrites, and biomedical materials). Author or co-author of more than hundred papers and two monographs, in the field of quantum electronics, laser technology and physics, biomedicine, telecommunications and computer technology. 